\title{
Aménagements agraires préhispaniques dans un milieu volcanique : Analyses des données LiDAR du Malpaís de Zacapu, Mexique
}

\author{
Pre-Hispanic agrarian developments in a volcanic environment: Analysis \\ of LiDAR data from the Malpaís de Zacapu, Mexico
}

\author{
Antoine Dorison ${ }^{1}$ \\ ${ }^{1}$ Postdoctorant, UMR8096 ArchAm Archéologie des Amériques (CNRS), antoine.dorison@gmail.com \\ MAE, Laboratoire ArchAm, 21 allée de l'université 91000 Nanterre
}

RÉSUMÉ. La multiplication récente de couvertures LiDAR en Mésoamérique (Mexique et Guatemala) révèle peu à peu l'ampleur jusqu'ici sous-estimée de l'aménagement des espaces à l'époque préhispanique. Les sites d'habitat connus s'avèrent intégrés dans des paysages intensément modifiés, avant tout pour être cultivés, soulignant une fois de plus l'importance de l'agriculture dans ces sociétés sans animal de trait ni bétail. Inscrit dans cette mouvance, cette contribution présente le cas probant du LiDAR du Malpaís de Zacapu $\left(90 \mathrm{~km}^{2}\right)$, dans l'Ouest mexicain, en s'intéressant plus spécialement aux aménagements agraires. L'étude combine terrain et imagerie numérique (LiDAR, imagerie satellite) pour aborder l'aménagement anthropique de ce milieu volcanique caractérisé par des coulées de lave chaotiques où la couverture pédologique est plus ou moins développée. Elle convoque les méthodes de l'archéogéographie et de la géopédologie appliquée pour mettre à jour la cartographie des vestiges archéologiques et des sols de la zone. Les nombreuses structures découvertes bouleversent notre vision de la chronologie d'occupation locale. Parallèlement, l'étude met en lumière des aménagements agraires adaptés aux spécificités des formes du terrain et aux types de sols. Elle met finalement en exergue des choix culturels pour la sélection des espaces cultivés qui évoluent en fonction des époques.

ABSTRACT. Recent LiDAR-based surveys in Mesoamerica (Mexico and Guatemala) slowly reveal the underestimated magnitude of prehispanic landscape management. Known residential sites prove to be integrated within deeply modified landscapes, primarily to be cultivated, thus highlighting once more the importance of agriculture in these societies deprived of beasts of burden or cattle. Consistent with this research trend, this contribution presents the compelling case study of the Malpaís de Zacapu LiDAR $\left(90 \mathrm{~km}^{2}\right)$, in West Mexico, focusing on agrarian features. The study combines field work and digital imagery (LiDAR, satellite imagery) to address landscape modification in this volcanic environment characterized by chaotic lava flows displaying little or no soil cover. We used methods from archaeogeography and applied geopedology to update the archaeological and soil maps of the area. The numerous remains brought to light dramatically change our understanding of local settlement history. In the meantime, the study underlines agrarian features adapted to specific landforms and soil types. It finally points out that the selection of cultivated areas is also governed by cultural choices that have changed through time.

MOTS-CLÉS. Occident du Mexique, agriculture préhispanique, LiDAR, archéogéographie, géoarchéologie, pédologie, géomorphologie.

KEYWORDS. West Mexico, prehispanic agriculture, LiDAR, archaeogeography, geoarchaeology, soil science, geomorphology.

\section{Introduction}

L'agriculture constituait la base de l'économie des peuples mésoaméricains préhispaniques (Rojas Rabiela 1988; Whitmore et Turner 2001). Deux différences majeures la distinguent cependant de celle de l'Ancien Monde : l'absence d'animal de trait et l'importance réduite de l'élevage, limité au dindon, au chien et à l'abeille. Les paysages agraires de Mésoamérique ont été façonnés manuellement. La manipulation de sol était généralisée. De nombreux aménagements (ex. terrasses, canaux...) en constituent aujourd'hui les vestiges matériels (Donkin 1979). Bien qu'ils soient documentés de longue date (Acuña 1987 [c. 1570-80] : 104), leur ampleur spatiale a jusqu'ici été sous-estimée. La multiplication des couvertures LiDAR au Mexique et au Guatemala (Fisher et al. 2011 ; Chase et al. 
2012 ; Canuto et al. 2018; Pereira et Padilla 2018; Dorison 2019 entre autres) révèle aujourd'hui l'aménagement anthropique quasiment continu des milieux physiques pour l'exploitation agricole autour et à l'intérieur des sites d'habitat.

Cette contribution présente le cas du LiDAR du Malpaís de Zacapu, dans l'Ouest du Mexique. Le Malpaís est un ensemble géologique caractérisé par la superposition de coulées de lave récentes, pléistocènes et holocènes, réparties sur environ $50 \mathrm{~km}^{2}$ (Demant 1992 ; Reyes et al. 2018 ; Maghoub et al. 2018). Ce «mauvais pays » de Zacapu est actuellement réputé impropre à la mise en culture à cause de l'omniprésence des affleurements rocheux. Néanmoins, la zone est bien connue archéologiquement, car les épanchements volcaniques les plus jeunes et chaotiques (1525-1420 av. J.-C., 20080 av. J.-C. et 830-960 apr. J.-C.), partiellement ou complètement dépourvus de couverture pédologique, ont été aménagés au milieu du XIII ${ }^{\mathrm{e}}$ siècle pour la construction de quatre établissements à caractère urbain (Michelet et al. 2005; Forest 2014). Hormis des investigations archéologiques intrasites conduites dans le cadre de plusieurs projets français successifs (Michelet 1992; Darras 1998 ; Pereira et Padilla 2018), les connaissances demeuraient limitées en ce qui concerne l'inscription de ces établissements au sein de la microrégion et les modalités de la subsistance des populations urbaines. Pour combler ces lacunes, une étude de l'environnement des centres urbains a été mise en place dès 2013 pour identifier les traces d'occupation et évaluer le potentiel agronomique des sols (Dorison 2019; Dorison et Michelet 2015; Pereira et al. 2016). Elle a efficacement été complétée en 2015 par une opération LiDAR de $90 \mathrm{~km}^{2}$ couvrant l'ensemble du Malpaís. L'imagerie numérique et les travaux de terrain ont mis au jour de nombreux vestiges, chronologiquement plus anciens ou contemporains des sites urbains et relevant majoritairement de la sphère agricole. La présente contribution se focalise sur les aspects techniques de cette recherche.

\section{Contexte environnemental}

À $300 \mathrm{~km}$ à l'ouest de Mexico au nord de l'actuel Michoacán, la région de Zacapu est subtropicale tempérée (García 2004) (figure 1). La mousson apporte $800 \mathrm{~mm}$ d'eau par an concentrés entre mai et novembre. À $2000 \mathrm{~m}$ d'altitude, elle se place au cœur de la Ceinture Volcanique Transmexicaine (Hasenaka et Carmichael 1985) et du Champ Volcanique Michoacán Guanajuato (CVMG) (Siebe et al. 2014). Le paysage zacapense est ponctué d'édifices volcaniques dont une majorité de cônes formés au cours d'éruptions uniques (monogénétiques), desquels sont issues les coulées miocènes à holocènes qui constituent l'essentiel du substrat géologique (Hasenaka et Carmichael ibid. ; Demant 1992; Reyes et al. 2018). Des éruptions historiques dans le CVMG, comme celle du Paricutín (1943-52; Pioli et al. 2008), témoignent de son activité continue. Récemment, des éruptions contemporaines de l'occupation préhispanique ont été datées au sud de notre région d'étude (El Metate, c. 1250 apr. J.-C.; Chevrel et al. 2016) et au sein du Malpaís de Zacapu (El Malpaís Prieto, 830-960 apr. J.-C.; Maghoub et al. 2018). Zacapu se caractérise en outre par son bassin lacustre. Fruit de l'activité volcanique et tectonique quaternaire (Tricart 1992 ; Ferrari et al. 2012), il est fermé à l'ouest par le Malpaís. Le lac originel a été drainé pour la mise en culture autour de 1900 via le surcreusement de son exutoire temporaire au nord-ouest du bassin (Arnauld et al. 1993). La fenêtre d'étude du présent travail a été définie dans cette zone, à cheval entre la plaine lacustre et les hautes terres volcaniques. 


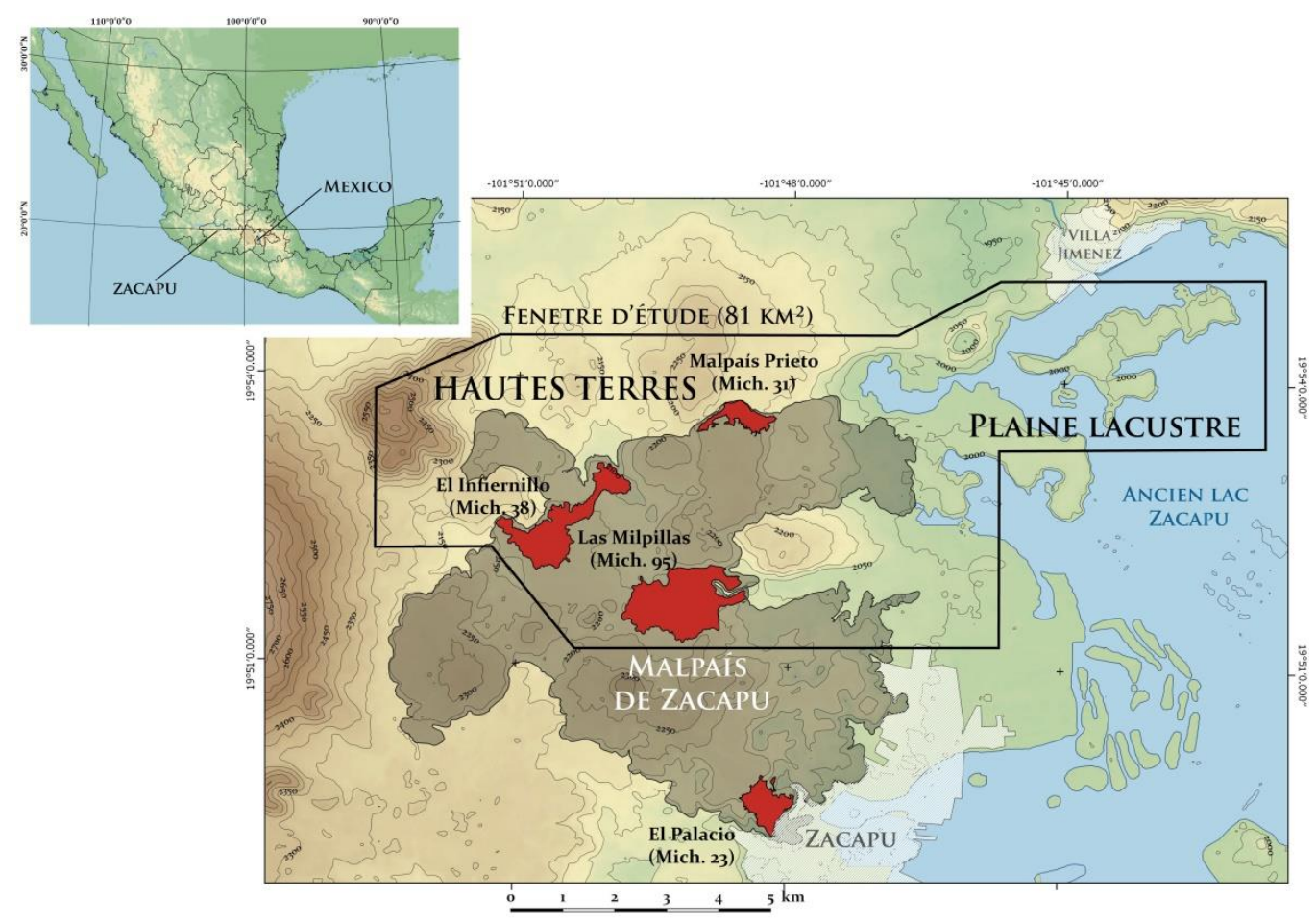

Figure 1. Localisation générale de l'étude sur le territoire mexicain et par rapport au bassin versant de Zacapu (C) A. Dorison.

En ce qui concerne la pédologie, outre l'altération des roches volcaniques, les dépôts de cendre constituent le matériau parental de la majorité des sols (DETENAL 1979; Dorison 2019). De par la sensibilité à l'érosion de ces contextes géomorphologiques, nombre de sols se sont aussi développés sur des colluvions ou des alluvions. Dans la plaine, l'ancien lac a conduit à la formation de sols lacustres (tourbes en voie d'humification), encore influencés aujourd'hui par la faible profondeur de la nappe phréatique. La couverture pédologique est donc variée : les sols humides de la plaine (Histosols, Gleysols, Vertisols, Phaeozems) laissent place à des sols téphriques dans les hautes terres volcaniques et le Malpaís (Andosols, Cambisols, Phaeozems, Luvisols, Vertisols), au sein desquels l'humidité est rapidement drainée en raison de la perméabilité du substrat andésitique. La faible épaisseur des solums, voire l'absence de sol, est finalement caractéristique des coulées les plus récentes (Pléistocène récent et Holocène, moins de 100000 ans) (Leptosols). L'excès d'humidité est le principal enjeu agronomique de la plaine. Son manque est celui des hautes terres.

En conséquence, le couvert végétal et l'usage actuel des sols sont eux aussi variés (Labat 1992). La plaine est caractérisée par une agriculture extensive partiellement mécanisée qui tire profit du niveau de la nappe phréatique par l'ouverture/fermeture de l'exutoire du bassin (Gougeon 1991). Les hautes terres sont divisées entre les coulées anciennes (plus de 100000 ans), où l'agriculture pluviale côtoie des plaines de pâture pour les bovins, et les coulées plus récentes couvertes de forêts de chêne, dont certaines servent aussi de pâturage. C'est sur ces dernières, considérées comme des malpaíses, qu'ont principalement été reconnus les sites archéologiques, protégés des labours, tractés par l'abondance des affleurements et cachés par la végétation.

\section{Recherches archéologiques permettant l'identification des structures sur LiDAR}

La fenêtre d'étude regroupe trois des quatre centres urbains du Malpaís de Zacapu: Mich. 31 (Malpaís Prieto), Mich. 38 (El Infiernillo) et Mich. 95-96 (Las Milpillas). Ce dernier fut le premier fouillé et partiellement cartographié à l'alidade dans les années 1980 (Puaux 1989, Migeon 1998, Michelet 1998). Les trois centres urbains ont ensuite fait l'objet d'une cartographie de densité de structures effectuée à pied dans les années 1990 (Michelet ibid.). Quelques sondages, nettoyages et enregistrements topographiques ont alors été conduits sur Mich. 38. Les années 2010 ont vu le 
développement d'investigations focalisées sur Mich. 31, comprenant la fouille de divers espaces (domestiques, funéraires, cérémoniels) (Forest 2014; Manin 2015; Jadot 2016; Pereira et Padilla 2018) et une cartographie au GPS de l'intégralité du site. À la même époque, un enregistrement GPS a aussi été réalisé dans des parties de Mich. $95-96$ et 38 (Forest ibid.). Au total, Mich. 31 comptait alors plus de 2500 structures enregistrées, Mich. 38 plus de 1200 et Mich. 95-96 plus de 500 (Forest ibid. : 168, 146, 153). Aux environs des centres urbains, n'étaient alors connus que cinq sites secondaires dans les hautes terres et sept dans la plaine lacustre, ne comptant chacun qu'une vingtaine à une cinquantaine de structures (Migeon 1998, 2016). Un unique sondage avait été réalisé (Mich. 68) et seuls trois établissements bénéficiaient de croquis partiels (Mich. 32, 34-70 et 68). Le reste des sites n'avait fait l'objet que de visites succinctes avec description de l'organisation spatiale et ramassages de matériel en surface.

La datation des espaces reposait et repose toujours principalement sur une typochronologie céramique (Michelet 2013; Jadot 2016). L'ancrage chronologique est assuré par un corpus originel de 22 dates radiocarbone (Michelet 1992), récemment augmenté de 15 nouvelles dates (Pereira et al. sous presse). Les centres urbains sont issus d'une colonisation humaine secondaire et caractérisent le Postclassique moyen (1250-1450 apr. J.-C.) (Michelet et al. 2005). Aux époques antérieures (Classique : 200-600 apr. J.-C., Épiclassique : 600-900, et Postclassique ancien : 900-1250), des populations évoluent dans la région, aux abords du lac puis dans les hautes terres (Arnauld et Faugère 1998). Pourtant, avant notre investigation microrégionale, le Nord-Ouest du bassin - dont le Nord du Malpaís - était considéré comme un no man's land où ces époques anciennes étaient très mal représentées et documentées.

L'ensemble de ces opérations archéologiques a fourni une première typologie des structures construites sur laquelle s'appuyer (voir notamment Forest $2014: 163-248$ ), tant pour les identifications de terrain que pour l'exploitation du LiDAR. Les régions adjacentes - versant du Rio Lerma (Faugère 1996; Darras 1999), sud de l'État de Guanajuato (Cardenas García 1999; Pereira et al. 2005), bassin de Pátzcuaro (Gorenstein et Pollard 1983 ; Fisher et Leisz 2013) - offraient des corpus de comparaison.

Bâtiments cellulaires. Malgré quelques rares exceptions, la plupart des bâtiments sont monocellulaires. De leurs murs à parement simple ne subsistent que les premières assises en pierres sèches non équarries. Ils étaient surmontés de parois en adobe et d'un toit en chaume aujourd'hui disparus. En plan, ces structures sont quadrangulaires (ex. habitats, bâtiments de réunion, annexes domestiques) ou rondes (ex. base de greniers), à quelques exceptions près. De manière générale, les habitats de l'Épiclassique sont plus petits que ceux du Postclassique moyen (Dorison 2019).

Plateformes. De nombreuses plateformes et terrasses résidentielles sont construites en pierres sèches ou en terre avec contention en pierre. Les formes et plans sont variés mais généralement plus ou moins nettement orthonormés.

Soubassements pyramidaux. Ils sont constitués d'un empilement de plateformes en pierre de plus en plus réduites en gradins. Dès les premiers temps des recherches, une distinction a été faite entre les pyramides carrées de l'Épiclassique et celles rectangulaires du Postclassique moyen.

Places surbaissées. Inscrites dans une tradition de l'Occident mexicain (Cardenas García 1999), des places surbaissées rectangulaires associées à l'Épiclassique, dites patios hundidos, sont documentées dans la région étendue (Arnauld et al. 1993) mais pas dans le Malpaís.

Terrains de jeu de balle. Typiquement mésoaméricains, les terrains de jeu de balle sont aussi associés à l'Épiclassique (Taladoire 1989). Ils se composent de deux plateformes rectangulaires allongées encadrant un espace plan central rectangulaire également. Des aires terminales peuvent leur donner un plan en $\ll \mathrm{I} »$. 
Éléments linéaires. Les aménagements linéaires constituent la dernière grande catégorie de structures construites. Outre les murs isolés, ils sont principalement représentés par les terrasses agricoles. Jusqu'à récemment, elles étaient essentiellement connues sous la forme de simples alignements de pierres perpendiculaires à la pente pouvant créer un changement de niveau plus ou moins sensible.

\section{Méthodes cartographiques}

Deux méthodologies complémentaires ont été déployées pour réaliser une cartographie archéologique et géopédologique dans la fenêtre d'étude de $81 \mathrm{~km}^{2}$ focalisée sur le Nord-Ouest du bassin de Zacapu (figure 2): une approche archéogéographique (Chouquer 2008) et une étude géopédologique (Zinck 2012).
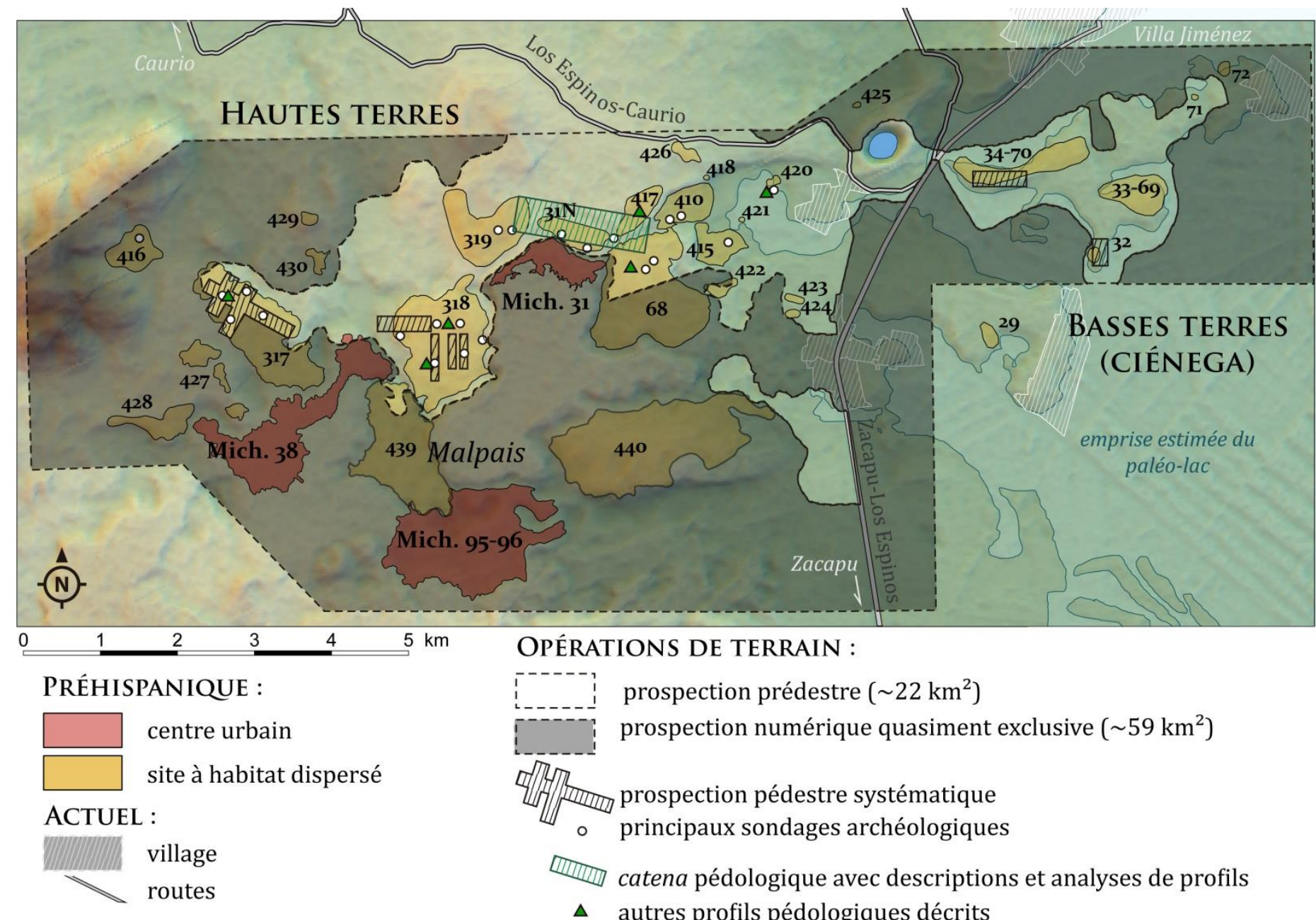

OPÉRATIONS DE TERRAIN :

Figure 2. Synthèse des opérations $\odot$ A. Dorison.

\section{Archeogéographie : prospections pédestres, cartographie GPS et LiDAR}

Pour mettre à jour la carte archéologique, nous avons d'abord procédé à des prospections pédestres sur environ $27 \%$ de la fenêtre $\left(22 \mathrm{~km}^{2}\right)$. Hors des forêts, elles ont été guidées par l'identification préalable d'anomalies de végétation sur images satellites. Sous couvert forestier, elles se concentraient systématisées dans les zones où des sites étaient documentés. Elles prenaient alors la forme de «battues » de cinq à six participants régulièrement espacés et guidés par deux récepteurs GPS le long d'un maillage virtuel de $100 \times 100 \mathrm{~m}$ calé sur la grille UTM. Les éléments rencontrés étaient enregistrés par un ou plusieurs points GPS, mesurés, dessinées, orientés, puis redessinés numériquement (numérisés) sur Qgis à l'aide d'une grille vecteur de $1 \times 1 \mathrm{~m}$. La datation des éléments est exclusivement basée sur la céramique. Celle-ci a été collectée en surface au cours des prospections, 
puis des structures ou groupes présentant un fort potentiel informatif ont été sélectionnés pour entreprendre des sondages de $2 \times 2 \mathrm{~m}^{1}$ en privilégiant les aménagements agraires.

Au printemps 2015, dans le cadre de l'ANR Mesomobile (2015-2018), un LiDAR de $90 \mathrm{~km}^{2}$ a été réalisé par le National Centre for Airborne Laser Mapping de Houston (NCLAM) (figure 3). Il a permis de construire un modèle numérique d'élévation (MNE) d'une résolution moyenne de $50 \mathrm{~cm}$. Il couvre $60 \%$ de la fenêtre d'étude. L'analyse de l'image brute a été optimisée par différents algorithmes préexistants : ombrages, classes de pentes, contours, ouvertures positive et négative (Yokoyama et al. 2002), skyview factor (SVF) (Böhner et Antonić 2009), local relief model (LRM) (Hesse 2010), etc. Les structures étaient directement identifiées et numérisées sur Qgis par carrés d'observation de $200 \times 200 \mathrm{~m}$. Plusieurs traitements furent systématiquement testés sur une même zone pour assurer la meilleure lecture possible. Notre investigation a notamment permis de mettre en place une superposition de rendus par transparence particulièrement efficace pour favoriser la lecture du LiDAR. Un ombrage multiple au contraste augmenté servait de toile de fond. Nous lui superposions le LRM (40\% de transparence) puis le SVF (70 \% de transparence) (4 sur figure 3).
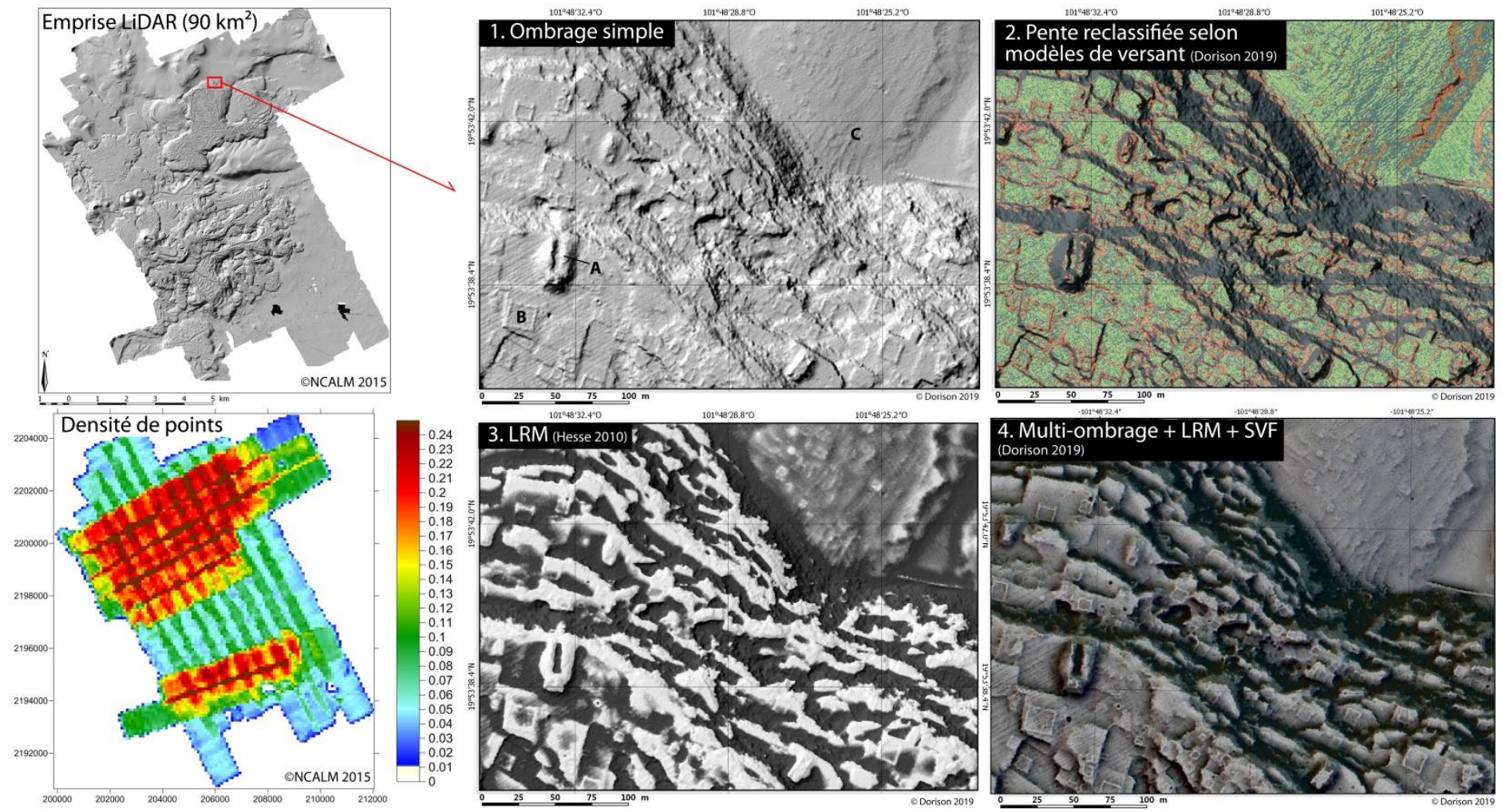

Figure 3. Emprise du LiDAR, densité du nuage de points et exemples de traitements $\subset$ A. Dorison. $A$ : pyramide pillée en son centre, $B$ : habitat d'élite, $C$ : réseau de terrasses agricoles.

Cette étape d'interprétation du LiDAR a en outre bénéficié de l'apport d'un exercice de saisie collaborative sur webgis à l'Université de Paris 1 , supervisé par L. Costa, G. Pereira, M. Forest et A. Dorison (Forest et al. sous presse). Deux ateliers d'un semestre ont été réalisés en 2016 (22 participants) et 2017 (6 participants). Après une présentation succincte du contexte culturel et des structures connues, les étudiants choisissaient un carré à numériser sur la grille de $200 \times 200 \mathrm{~m}$ préalablement intégrée au webgis développé par L. Costa sur la plateforme DynMap. Chaque étudiant avait un accès distant au webgis le temps de l'exercice et pouvait numériser en ligne autant de carrés que souhaité. Un état de l'avancée a été réalisé à mi-parcours et une correction globale en fin de semestre. Ces ateliers se sont focalisés sur les centres urbains de Mich. 38 et 95-96.

\footnotetext{
${ }^{1}$ La présente étude intègre les données céramiques de 27 sondages dans la fenêtre et les résultats d'opérations non publiées consultables dans les rapports du projet Uacúsecha (Dorison et Michelet 2015 ; Pereira et al. 2016).
} 
Pour la cartographie géopédologique, nous sommes partis des images satellites Google Earth pour délimiter les géoformes de la zone (coulées, cônes...), en parallèle de la consultation des cartes géologiques (Reyes et al. 2018), pédologique (DETENAL 1979) et géomorphologique (Tricart 1992) réalisées antérieurement. Ces découpages ont été affinés (sous-division en formes du terrain) par des vérifications in situ en surface et à l'aide d'une tarière manuelle Prückhauer qui permet d'obtenir une carotte de sol de $1 \mathrm{~m}$ pour un examen de base (100 carottages). Par la suite, 25 profils pédologiques ont été décrits, dont 15 ont été échantillonnés et analysés au laboratoire de pédologie de l'Université Nationale Autonome de Mexico (UNAM). Ces derniers ont été concentrés sur une coulée du Pléistocène moyen où des vestiges d'agriculture avaient été identifiés (figure 2). Les autres profils ont fourni des observations ponctuelles sur des coulées plus récentes et en bordure du paléo-lac.

Les traitements LiDAR ont permis de comparer les paramètres morphométriques et morphographiques des espaces entre eux. En plus de préciser ainsi les observations à pied, des extrapolations ont pu être faites entre les zones documentées sur le terrain et celles connues uniquement par les données numériques (LiDAR, satellites, photographies). Le LiDAR a aussi permis de simuler les flux et concentration théorique d'eau à la surface des géoformes. Nous avons utilisé les algorithmes de flux hydrique, de captation d'eau ou l'indice d'humidité topographique du logiciel SAGAgis (Olaya 2004). Sur cette base, nous avons proposé des caractéristiques morphogénétiques pour les formes du terrain non visitées in situ.

\section{Résultats}

\section{Réévaluation de l'occupation du Nord-Ouest du bassin}

Ces nouvelles recherches associant technologie LiDAR et travaux de terrain offrent une révision profonde de l'occupation dans la fenêtre d'étude. Le nombre des structures numérisées s'élève à plus de 12 000, soit au moins trois fois plus qu'avant l'acquisition du LiDAR. En outre, de nombreux espaces identifiés restent à cartographier. L'étude met en exergue une différence qualitative et quantitative en termes de vestiges entre les hautes terres intensément aménagées et la plaine lacustre quasiment vide. Malgré des biais taphonomiques et méthodologiques certains dans la plaine (destructions liées à l'agriculture coloniale et moderne, possible enfouissement des éléments sous colluvions, alluvions et sédiments lacustres, absence partielle de couverture LiDAR pour cette zone), la différence avec les hautes terres est trop nette pour ne pas correspondre à un choix culturel.

La deuxième avancée majeure est la redéfinition de la chronologie locale. Un foyer de peuplement ancien d'une ampleur inédite a été mis au jour dans les hautes terres (Dorison et Michelet 2015; Pereira et al. 2016 ; Dorison 2019). Il se met en place à l'Épiclassique, entre le $\mathrm{VI}^{\mathrm{e}}$ et le $\mathrm{VII}^{\mathrm{e}}$ siècle, et dure 300 ans. L'habitat est alors concentré sur les affleurements des coulées du Pléistocène récent. La zone est désertée au $X^{\mathrm{e}}$ siècle. L'éruption du Malpaís Prieto entre 830 et 960 (Maghoub et al. 2018) a influencé cet abandon. La recolonisation du Malpaís et son urbanisation dès 1250 (Michelet et al. 2005) ne sont pas remises en question mais la découverte de plusieurs sites secondaires montre que les aménagements de surface s'étendent au-delà des limites des centres urbains, notamment sur les coulées du Pléistocène moyen. Enfin, malgré l'abandon généralisé au $\mathrm{XV}^{\mathrm{e}}$ siècle, l'étude a révélé de façon inédite le maintien discret de l'occupation au nord-est du Malpaís pendant le Postclassique récent (1450-1522 apr. J.-C.) et la construction d'un village espagnol dès 1540 (Lefebvre et Quezada 2016).

\section{Réévaluation de la répartition des sols cultivables}

La cartographie géopédologique établit des différences d'ordre agronomique entre les coulées volcaniques au sein du Malpaís, trop souvent perçu comme une entité monolithique (figure 4). D’un point de vue théorique et dans une perspective d'agriculture préhispanique, les sols téphriques de 
moins de 100000 ans sont les plus intéressants dans les hautes terres. Caractéristiques des coulées du Pléistocène récent et des zones de colluvions, ils sont chimiquement fertiles, bien drainés, aérés, et facilement manipulables, car légers. Les principaux enjeux pour leur exploitation sont leur drainage rapide et leur sensibilité à l'érosion dans les pentes. Toutefois, leur porosité assure le maintien d'humidité résiduelle dans les dépressions. Enfin, dans les coulées volcaniques, l'abondance des affleurements rocheux qui pose problème aux paysans actuels utilisant des charrues, avait peu d'importance pour l'agriculture préhispanique manuelle. Les sols d'altération ancienne (de plus de 100000 ans) riches en argiles gonflantes sont plus difficiles à travailler (dessiccation/humectation en fonction des saisons). Ils caractérisent les coulées du Pléistocène ancien. L'excès d'humidité dans les dépressions peut par ailleurs être préjudiciable. De manière plus marquée, ces mêmes problèmes apparaissent dans les sols pourtant fertiles des anciennes rives de la plaine lacustre, à cause de la faible profondeur de la nappe phréatique. Un drainage efficace est nécessaire pour les exploiter. En somme, les hautes terres semblent donc plus propices à l'agriculture précolombienne que la plaine. Ce constat théorique est matérialisé par les nombreux aménagements agraires préhispaniques mis en lumière.

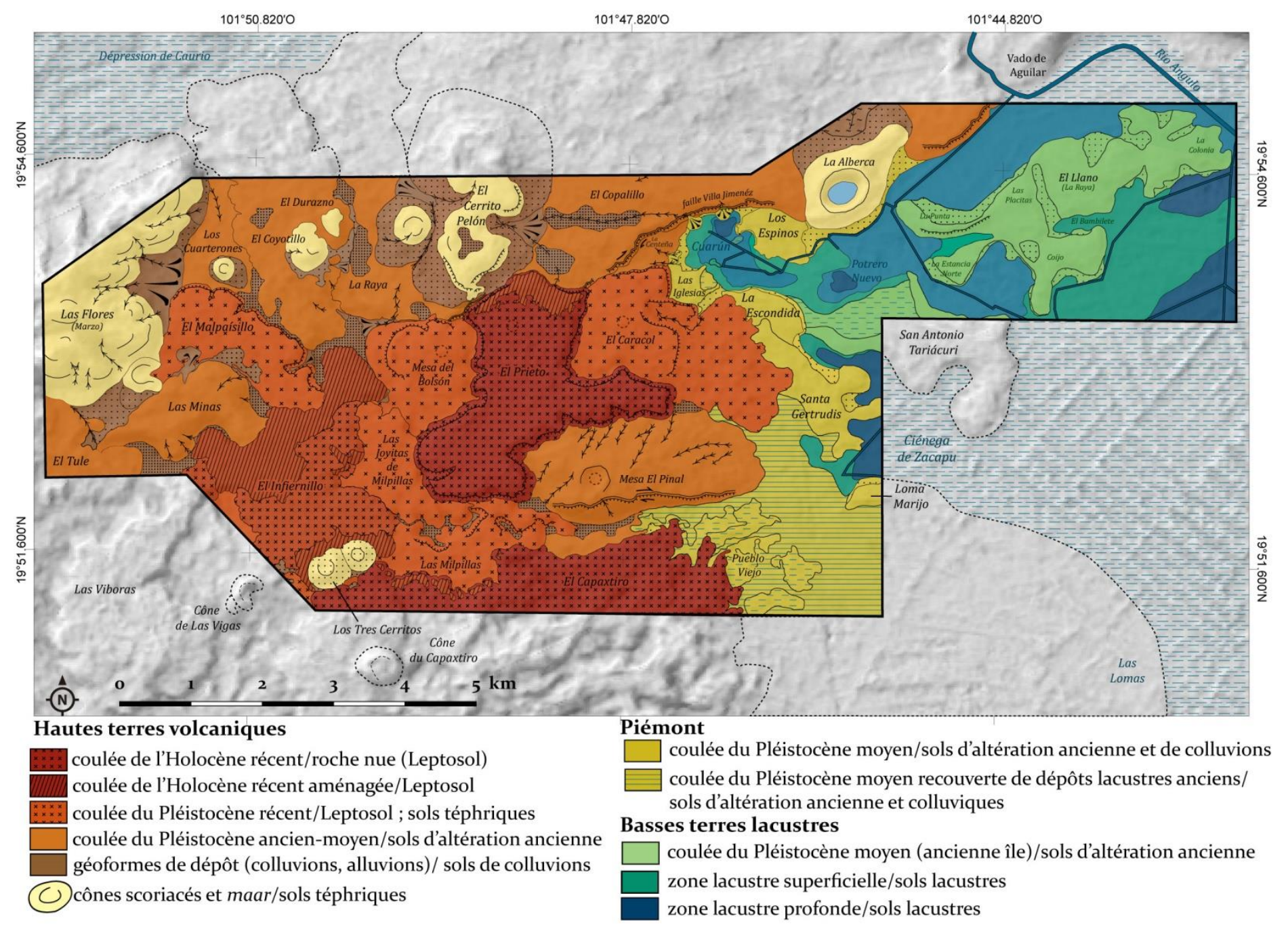

Figure 4. Carte géopédologique simplifiée de la fenêtre d'étude $\odot$ A. Dorison.

\section{Interprétation et discussion}

\section{Typologie des aménagements agraires}

La majorité des structures identifiées est associée à l'agriculture. La précision des données LiDAR mise en regard des travaux de terrain a permis d'établir une typologie détaillée de ces aménagements (figures 5 et 6). Tous sont construits pour tirer profit de sols spécifiques tout en palliant les contraintes agronomiques de leur contexte géomorphologique. 


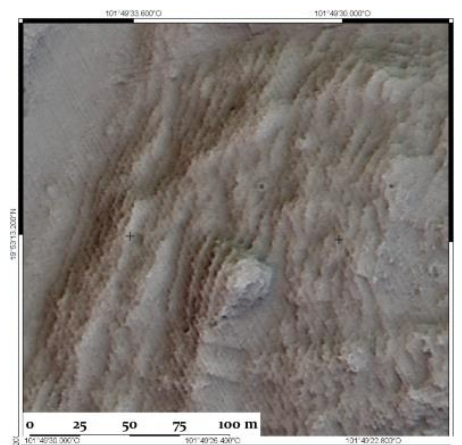

Terrasses de nivellement étroites et larges
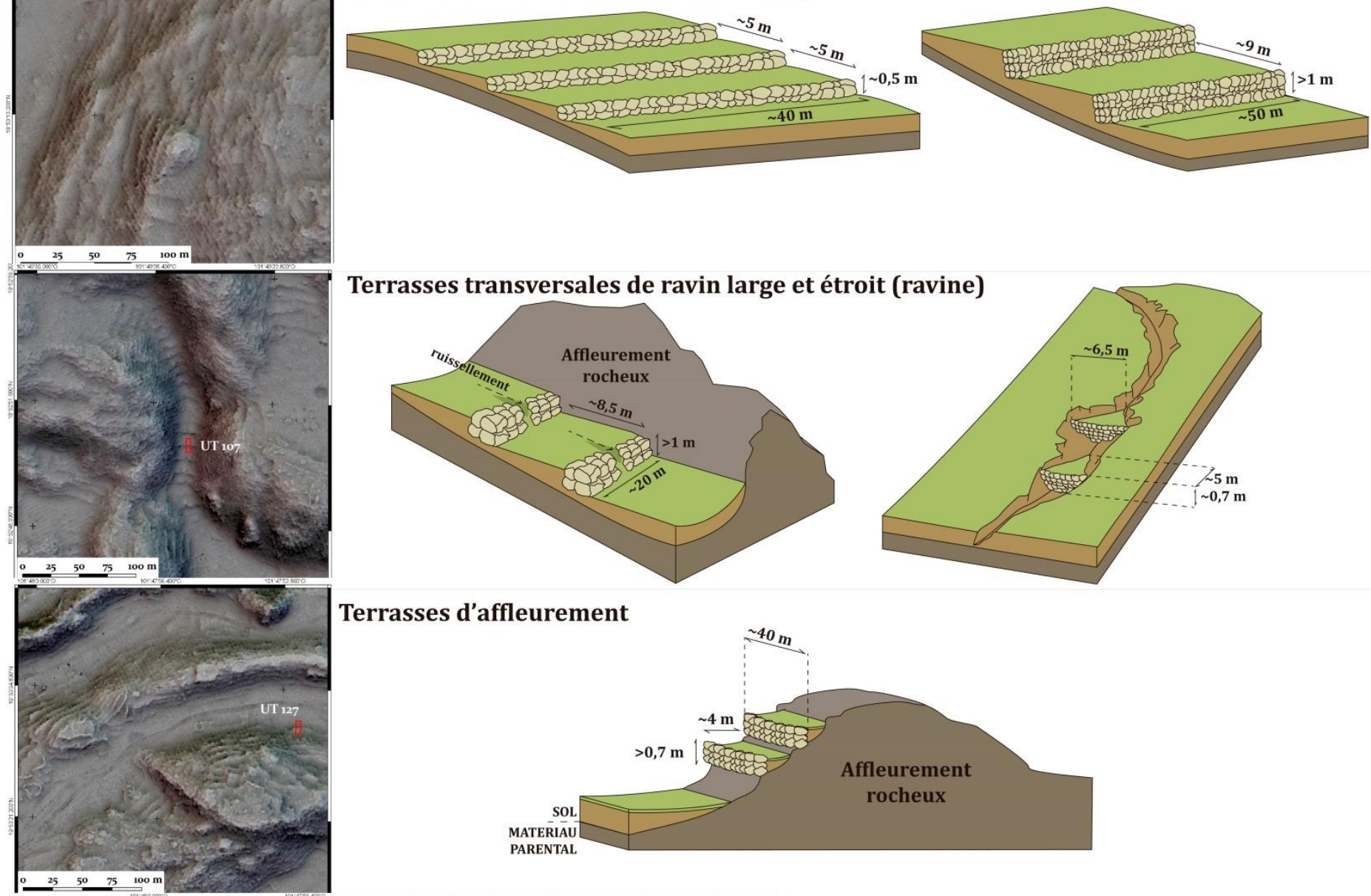

Terrasses transversales de ravin large et étroit (ravine)
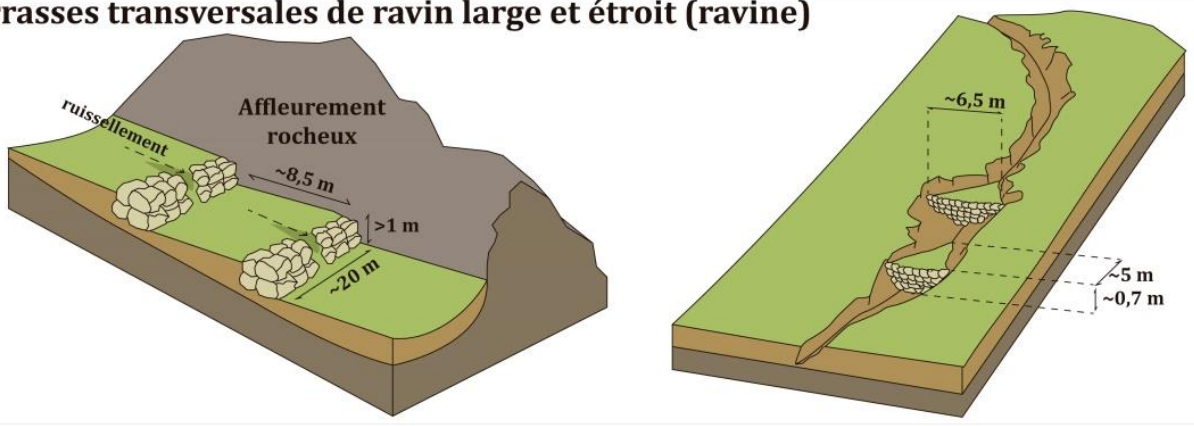

Terrasses d'affleurement
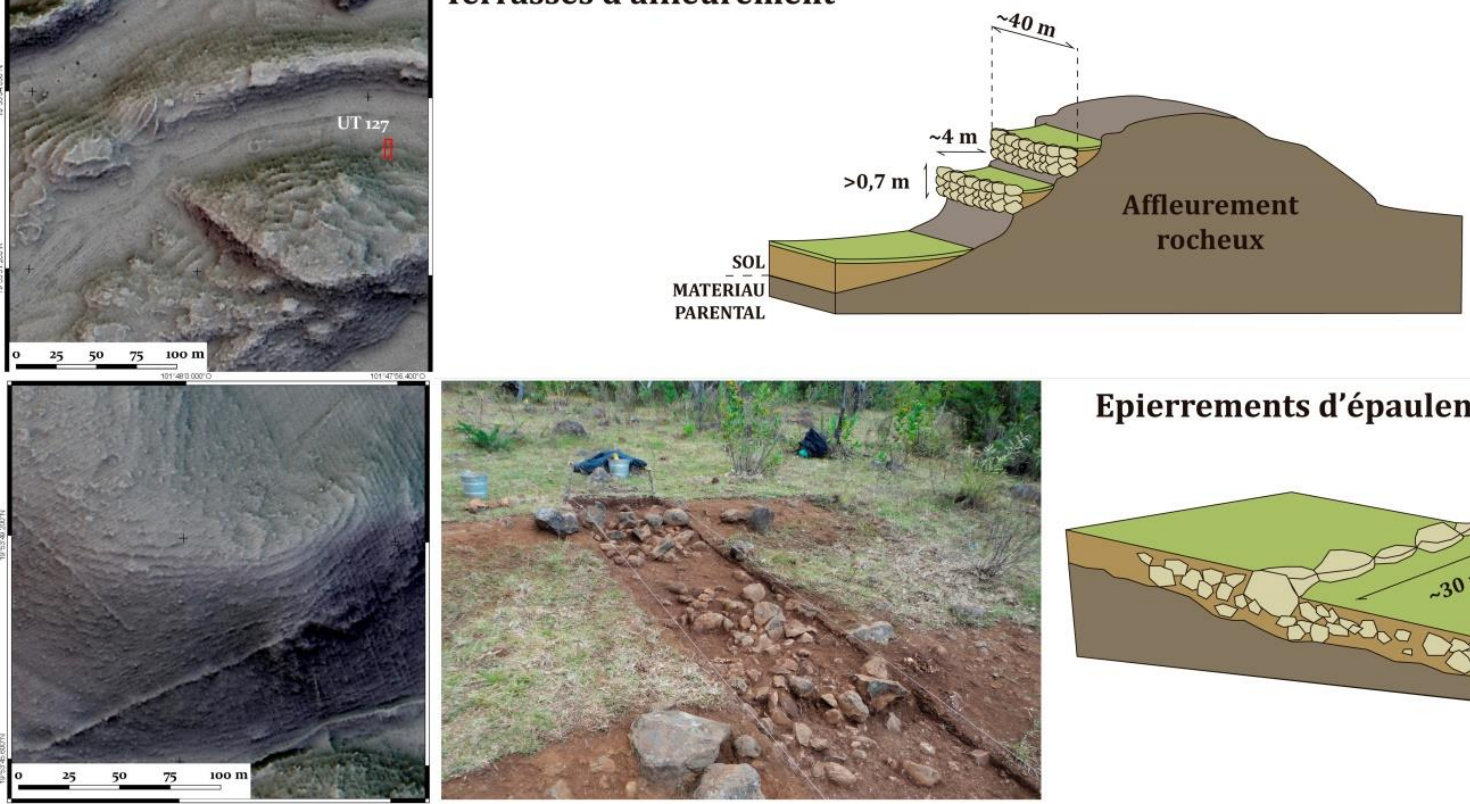

Epierrements d'épaulement

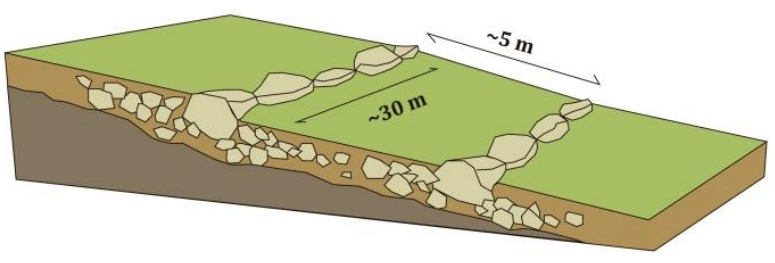

Figure 5. Typologie des aménagements agraires 1 : aménagements de zones en pente $\subset$ A . Dorison. 


\section{Epierrements/dégagements de zone chaotique}
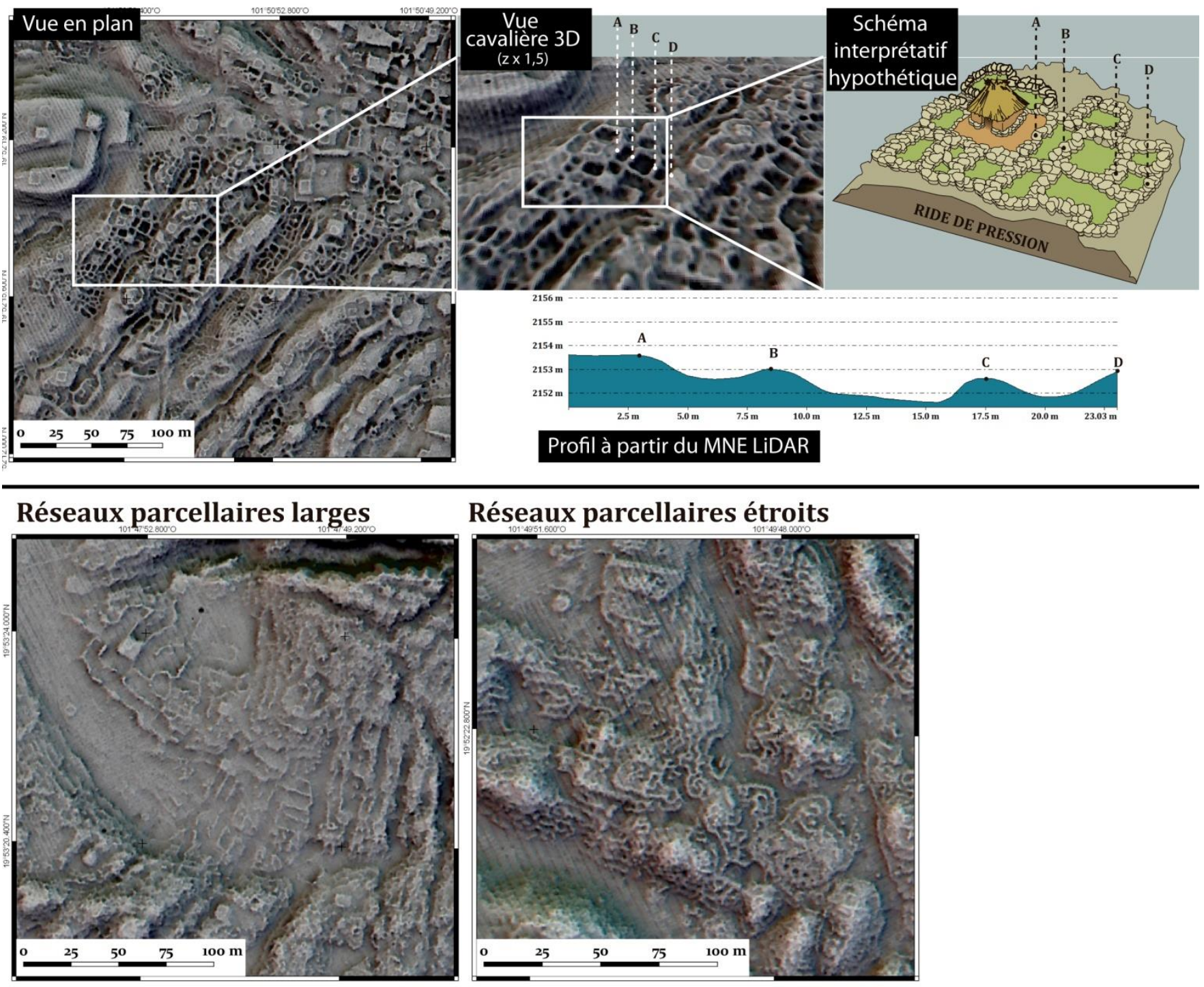

Figure 6. Typologie des aménagements agraires 2 : aménagements de zones planes $\subset$ C $A$. Dorison.

Les terrasses. Majoritaires, nous en définissons quatre grands types.

Les terrasses de nivellement (étroites ou larges selon l'espacement) ont un profil en escalier. Elles se développent sur des versants droits ou convexes à sols téphriques, principalement sur les coulées du Pléistocène récent. Les réseaux denses sont généralement rectilignes, voire orthonormés, mais peuvent aussi épouser la courbure du versant. Elles limitent l'érosion, créent un solum plus épais et ralentissent les déperditions d'eau dans la pente.

Les terrasses transversales de ravin (ou ravine) exploitent des sols téphriques, de colluvions et d'alluvions. Barrant la pente des ravins, elles permettent de retenir les flux de sol et d'eau naturellement concentrés vers le talweg. Les solums sont épaissis et le drainage interne modérément freiné.

Les terrasses d'affleurement se répartissent le long des rides de pression des coulées volcaniques du Pléistocène récent. Le sol est accumulé manuellement en amont du mur de contention pour créer un espace cultivable exigu, souvent proche de structures d'habitat qui occupent le sommet des affleurements.

Les zones de suintement ou seepages (zones de faible pente en amont des épaulements où le drainage interne est lent et latéral [Dalrymple et al. 1968]) et pieds de versant sur sols téphriques ou de 
colluvions sont aménagés de terrasses spécifiques, isolées ou en réseaux plus lâches que les terrasses de nivellement. Elles retiennent la terre et l'humidité.

Les épierrements d'épaulements. Sur les coulées du Pléistocène moyen, certains épaulements à sols peu épais, argileux et pierreux présentent des aménagements qui, en plan, ressemblent à des terrasses. Organisés en réseaux serrés épousant la courbure du versant, ils n'en modifient que légèrement le profil (pas d'escalier). En surface, seuls des alignements de pierres sont visibles. La fouille indique que leur construction procède d'épierrements puis de concentration des blocs, impliquant d'importants remaniements des horizons de surface. Ils tirent profit de l'humidité conservée dans les argiles et du gradient thermique des pierres, qui limite les écarts de température dans le sol (Gras 1994).

Les aménagements de zones chaotiques planes. Le LiDAR permet d'identifier d'autres aménagements à la morphologie inédite dont la fonction est peut-être agricole. On les observe à la surface de certaines coulées volcaniques holocènes où une couverture pédologique superficielle s'est développée. En plan, ils se présentent comme des groupes de cuvettes contigües mesurant chacune entre une dizaine de mètres carrés et plusieurs hectares. Leur morphologie obéit vraisemblablement à un système d'épierrements pour dégager des zones planes. Les rares visites de terrain indiquent que du sol est accumulé au fond des cuvettes dont les murs ne dépassent pas $1 \mathrm{~m}$ de haut pour plus de $1,5 \mathrm{~m}$ de large. Ces épierrements/dégagements s'organisent en réseaux tortueux sur de grandes surfaces. Le site urbain de Mich. 38 en compte plusieurs milliers répartis sur près de 100 ha. Sans fouille, nous ne pouvons être catégoriques quant à leur vocation agricole. C'est toutefois l'hypothèse la plus probable au vu du contexte géopédologique et dans la mesure où aucune autre fonction potentielle ne semble en adéquation avec leur morphologie (habitat, stockage, culte, voirie...).

Les limites parcellaires. Enfin, des limites parcellaires plus classiques sont construites dans les zones planes et chaotiques des coulées du Pléistocène récent. Ces murs sont plus larges que ceux des habitats $(>1 \mathrm{~m})$. Certains sont quasiment orthogonaux en plan mais la plupart présentent une organisation tortueuse.

Par ailleurs, aucun indice clair d'aménagement agraire n'a pu être observé sur les rives de l'ancien lac.

\section{Évolution des paysages agraires}

La fouille et les ramassages permettent de dater certains aménagements agraires. Par ailleurs, certaines structures à la morphologie bien documentée (pyramides, patios hundidos...) sont chacune caractéristiques d'une seule époque. Ainsi, l'analyse des structures identifiées sur les images LiDAR permet de proposer une typochronologie des espaces. Il s'agit d'identifier les récurrences dans l'organisation spatiale des zones agraires par rapport aux zones résidentielles et/ou civiques, où les structures sont mieux documentées et datées. Par exemple, une logique spatiale apparait entre les terrasses de nivellement et les zones qui présentent des patios hundidos, des jeux de balle et des plateformes monumentales typiques de l'Épiclassique. Les épierrements d'épaulements sont quant à eux cohérents avec l'organisation des groupes domestiques du Postclassique moyen.

Plus largement, il est donc possible de mettre en exergue une évolution chronologique dans la sélection des espaces cultivés et dans les contextes géopédologiques recherchés selon les époques. Les sols téphriques des coulées du Pléistocène récent sont favorisés à l'Épiclassique. On les aménage en construisant des terrasses de nivellement et des terrasses transversales de ravin. Les agriculteurs du Postclassique moyen exploitent plus généralement les sols d'altération ancienne des coulées du Pléistocène moyen. Certains sols téphriques continuent d'être cultivés mais d'autres sont délaissés, peut-être pour des raisons culturelles (territorialité entre les centres urbains, voir Dorison 2019). 


\section{Conclusion}

Le LiDAR montre que l'agriculture préhispanique zacapense a conduit à aménager de façon quasiment continue les hautes terres volcaniques autour et au cœur des sites d'habitat. Ce constat s'inscrit dans la lignée des découvertes récentes faites grâce à cette technologie dans le monde maya (Canuto et al. 2018) et indique que la forte emprise des sociétés sur leur environnement ne se cantonne donc pas aux milieux tropicaux humides de Mésoamérique. L’ampleur spatiale des aménagements atteste par ailleurs la capacité d'adaptation aux formes du terrain et aux types de sols dont les populations du Nord-Ouest du bassin de Zacapu ont fait preuve. L'analyse fine des données LiDAR, couplées aux approches de terrain, permet donc de saisir les paysages dans leur profondeur chronologique. Elle souligne des choix culturels (intérêt plus grand pour les sols de hautes terres au détriment, semble-t-il, des rives lacustres) et une évolution des pratiques selon les époques. Enfin, notre étude témoigne des connaissances empiriques en géomorphologie et en pédologie chez les agriculteurs préhispaniques qui font échos aux études actuelles d'ethnopédologie menées au Michoacán chez leurs descendants (Barrera et al. 2006).

\section{Bibliographie citée}

Acuña R. (ed.) 1987. «Relaciones geográficas del siglo XVI : Michoacán », Instituto de investigaciones anthropológicas, serie Antropológica : 74, UNAM, Mexico.

Arnauld M.-C. \& B. Faugère 1998. "Evolución de la ocupación en el Centro-norte de Michoacán y la emergencia del Estado Tarasco". in Darras V. (coord.) Génesis, culturas y espacios en Michoacán. CEMCA, Mexico : 13-34.

Arnauld M.-C., P. Carot \& M.-F. Fauvet-Berthelot 1993. Arqueología de las Lomas en la cuenca lacustre de Zacapu, Michoacán, México. CEMCA, Mexico.

Barrera N., A. Zinck \& E. Van Ranst 2006. "Local soil classification and comparison of indigenous and technical soil maps in a Mesoamerican community using spatial analysis", Geoderma $135: 140-62$.

Böhner J. \& O. Antonić 2009. "Land-surface parameters specific to topo-climatology”, in T. Hengl \& H. Reuter (eds.), Geomorphometry - Concepts, Software, Applications, Developments in Soil Science, Volume 33, Elsevier : 195-226.

Canuto M.A., F. Estrada-Belli, T.G. Garrison, S.D. Houston, M.J. Acuña, M. Kováč, D. Marken, P. Nondédéo, L. AuldThomas, C. Castanet, D. Chatelain, C. Chiriboga, T. Drápela, T. Lieskovský, A. Tokovinine, A. Velasquez, J. Fernandez-Diaz \& R. Shrestha 2018. "Ancient Lowland Maya Complexity as Revealed by Airborne Laser Scanning of Northern Guatemala”, Science, 361.

Cardenas García E. 1999. El Bajío en el Clásico: análisis regional y organizacion política, El Colegio de Michoacán, Zamora.

Chase A. F., D. Z. Chase, C. T. Fisher, S. J. Leisz \& J. F. Weishampel 2012. "Geospatial revolution and remote sensing LiDAR in Mesoamerican archaeology", Proceedings of the National Academy of Sciences, 109(32) : 12916-21.

Chevrel M. O., C. Siebe, M. N. Guilbaud \& S. Salinas 2016. “The AD 1250 El Metate shield (Michoacán): Mexico's most voluminous Holocene eruption and its significance for archaeology and volcanic hazards", The Holocene 26(3) : 471-88.

Chouquer G. 2008. Traité d'archéogéographie, Errance, Paris.

Dalrymple J. B., R. J. Blong \& A. J. Conacher 1968. "A hypothetical nine-unit landsurface model”, Zeitschrift für Geomorphologie, N.F. 12(1) : 60-76.

Darras V. 1998 (coord.). Génesis, culturas y espacios en Michoacán, CEMCA, México.

1999. Tecnologías prehispánicas de la obsidiana: los centros de producción de la región de Zináparo-Prieto, Michoacán, México. CEMCA, México.

Demant A. 1992. "Marco Geológico regional de la laguna de Zacapu, Michoacán, México », in Michelet D. (coord.) «El Proyecto Michoacán 1983-1987: medio ambiente e introducción a los trabajos arqueológicos”. Cuadernos de estudios michoacanos 4 : 53-72. CEMCA, Mexico.

Dirección de Estadística del Territorio Nacional (DETENAL) 1979. Carta Edafológica E14A11 Zacapu, escala 1:50000, México. 
Donkin R. A. 1979. Agricultural Terracing in the Aboriginal New World, University of Arizona Press, Tucson.

Dorison 2019. Archéologie des systèmes agraires préhispaniques de la région de Zacapu, Michoacán, Mexique, VII -XV siècle apr. J.-C., Thèse de doctorat, Université de Paris 1, Paris.

Dorison A. \& D. Michelet 2015. "La Zona Cuenca Noroeste: el entorno de los grandes asentamientos del noroeste de la cuenca de Zacapu", in G. Pereira, D. Michelet, A. Dorison, V. Darras, B. Faugère, L.Barba, M. Stevanato, O. Quezada \& J. Blanca - Informe técnico sobre los trabajos de campo llevados a cabo en Malpaís Prieto y otros asentamientos de la región de Zacapu, Michoacán. Temporada 7 (2014-15), Informe en el Archivo Técnico de la Coordinación Nacional de Arqueología, INAH, Mexico : 52-124.

Faugère B. 1996. Entre Zacapu y Río Lerma : culturas en una zona fronteriza, CEMCA, Mexico.

Ferrari L, T. Orozco Esquivel, V. Manea \& M. Manea 2012. "The dynamic history of the Trans-Mexican Volcanic Belt and the Mexico subduction zone », Tectonophysics 522-23 : 122-49.

Fisher, C.T. \& S. E. Leisz 2013. "New Perspetives on Purépecha Urbanism Through the Use of LiDAR at the Site of Angamuco, Mexico", in D. Comer \& M. Harrower, Space Archaeology: Mapping Ancient Landscapes with Air and Spaceborne Imagery, Springer, New York : 191-202.

Fisher, C.T., S. Leisz \& G. Outlaw 2011. "Lidar - a valuable tool uncovers an Ancient City in Mexico", Photogramm. Eng. Remote Sens. 77, 962-967.

Forest M. 2014. L'organisation sociospatiale des agglomérations urbaines du Malpaís de Zacapu, Michoacán, Mexique [1250-1450 après J.-C.], Thèse de doctorat, Université de Paris 1, Paris.

Forest M., L. Costa, A. Combey, A. Dorison \& G. Pereira (sous presse). "Testing Web Mapping and Active Learning to Approach Lidar Data", in Advances in Archaeological Practice 0(0) (2019), Society for American Archaeology : 115.

García E. 2004. Modificaciones al sistema de clasificación climática de Köppen, Serie Libros 6, Instituto de Geografía UNAM, México.

Gorenstein S. \& H. P. Pollard 1983. "The Tarascan Civilisation: A Late Prehispanic Cultural System", Vanderbilt University Publications in Anthropology 28, Nashville.

Gougeon O. 1991. "El noroeste de Mic hoacán: un paisaje en busca de identidad", in C. Reyes \& O. Gougeon, Paisajes rurales en el Norte de Michoacán, cuaderno de estudios michoacanos 3, CEMCA, México : 53-101.

Gras R. 1994. Sols caillouteux et production végétale, Quae, Paris.

Hasenaka T. \& I.S.E. Carmichael 1985. "The cinder cones of Michoacán - Guanajuato, central Mexico: their age, volume and distribution, and magma discharge rate", Journal of Volcanology and Geothermal Resources, 25, Amsterdam : $105-124$.

Hesse R. 2010. "LiDAR-derived Local Relief Models: a new tool for archaeological prospection", Archaeological Prospection $17: 67-72$.

Jadot E. 2016. Productions céramiques et mobilités dans la région tarasque de Zacapu (MichMexiqueMexique), Continuités et ruptures techniques entre 850 et $1450 \mathrm{apr}$. J.-C., Thèse de doctorat non publiée, Université de Paris 1 Panthéon-Sorbonne, Paris.

Labat J.-N. 1992. "Fitogeografía de la región de Zacapu", in Michelet D. (coord.) El Proyecto Michoacán 1983-1987: medio ambiente e introducción a los trabajos arqueológicos. Cuadernos de estudios michoacanos 4, CEMCA, Mexico.

Lefebvre K. \& O. Quezada 2016. Las Iglesias (Mich. 415), in G. Pereira, D. Michelet, A. Dorison, B. Faugère, O. Quezada, K. Lefebvre, M. Forest, I. Medina, I. Barrientos, H. Goudiaby, L. Barba, J. Blancas, A. Ortiz \& C. Gillot 2016 - Informe técnico sobre los trabajos de campo llevados a cabo en Malpaís Prieto y otros asentamientos de la región de Zacapu, Michoacán. Temporada 8 (2015-16), Informe en el Archivo Técnico de la Coordinación Nacional de Arqueología, INAH, Mexico : 10-33.

Mahgoub A.N., N. Reyes Guzmán, H. Boehnel, C. Siebe, G. Pereira et A. Dorison 2018. "Paleomagnetic constraints on the ages of the Holocene Malpaís de Zacapu lava flow eruptions, Michoacán (México) : Implications for archeology and volcanic hazards", Holocene, SAGE Publications 28(2) : 229-45.

Manin A. 2015. Aspects matériels et symboliques de l'utilisation des animaux dans le nord de la Mésoamérique, entre le Classique et la Conquête (200-1521 apr. J.-C.), Thèse de doctorat non publiée, Muséum National d'Histoire Naturelle, Paris. 
Michelet D. 1992. "El centro-norte de Michoacán: caractericas generales de su estudio arqueologico regional", in Michelet D. (coord.) El Proyecto Michoacán 1983-1987 : medio ambiente e introducción a los trabajos arqueológicos. Cuadernos de estudios michoacanos 4 : 9-52 CEMCA, Mexico.

1998. "Topografía y prospección sistemática de los grandes asentamiento del malpaís de Zacapu : claves para un acercamiento a las realidades sociopolíticas", in Darras V. (coord.) Génesis, culturas y espacios en Michoacán. CEMCA, Mexico.

2013. "Cerámicas del Centro-Norte de Michoacán entre el Clásico y el Posclásico", dans C. Pomédio, G. Pereira et E. Fernández Villanueva (éd.), Tradiciones cerámicas del Epiclásico en el Bajío y regiones aledañas: cronología e interacción, BAR International Series, Archaeopress, Oxford.

Michelet D., G. Pereira \& G. Migeon 2005. "La llegada de los uacúsechas a la región de Zacapu, Michoacán: datos arqueológicos y discusión", in Manzanilla L. (ed.) Reacomodos demográficos del Clásico al Posclásico en el centro de México. UNAM-Instituto de investigaciones antropológicas, México.

Migeon G. 1998. "El poblamiento del Malpaís de Zacapu y sus alrededores, del Clásico al Posclásico”, in Darras (coord.), Génesis, culturas y espacios en Michoacán, CEMCA, Mexico : 35-45.

2016. Patrones de asentamiento del Malpaís de Zacapu (Michoacán, México) y de sus alrededores en el Posclsáico, Paris Monographs in American Archaeology 46, Archaeopress, Oxford.

Olaya, V. 2004. A gentle introduction to SAGA GIS, The SAGA User Group e.V., Gottingen, Germany.

Pereira G. \& E. F. Padilla (eds.) 2018. La Cuidad Perdida. Raíces de los soberanos tarascos, INAH/CEMCA, Mexico.

Pereira G., Forest M., Jadot E., \& V. Darras (sous presse). "Ephemeral cities? The longevity of the Postclassic Tarascan urban sites of Zacapu Malpaís and its consequences on the migration process", in M.-C. Arnauld, G. Pereira \& C. Beekman (eds.), Ancient Mesoamerican Cities: Populations on the move, University Press of Colorado, Denver, USA.

Pereira G., D. Michelet \& G. Migeon 2005. "Transformaciones demográficas y culturales en el Centro-Norte de México en vísperas del Posclásico: los sitios de Cerro Barajas (suroeste de Guanajuato)", in Manzanilla L. (ed.) Reacomodos demográficos del Clásico al Posclásico en el centro de México. UNAM-Instituto de investigaciones antropológicas, México.

Pereira G., D. Michelet, A. Dorison, B. Faugère, O. Quezada, K. Lefebvre, M. Forest, I. Medina, I. Barrientos, H. Goudiaby, L. Barba, J. Blancas, A. Ortiz \& C. Gillot 2016. Informe técnico sobre los trabajos de campo llevados a cabo en Malpaís Prieto y otros asentamientos de la región de Zacapu, Michoacán. Temporada 8 (2015-16), Informe en el Archivo Técnico de la Coordinación Nacional de Arqueología, INAH, Mexico.

Pioli, L., E. Erlund, E. Johnson, K. Cashman, P. Wallace, M. Rosi \& H. D. Granados 2008. "Explosive dynamics of violent Strombolian eruptions: the eruption of Parícutin Volcano 1943-1952 (Mexico)", Earth and Planetary Science Letters, 271(1-4) : 359-368.

Puaux, O. 1989. Les pratiques funéraires tarasques (approche archéologique et ethnohistorique), Thèse de doctorat, Université de Paris 1, Paris.

Reyes N., C. Siebe, M. O. Chevrel, M. N. Guilbaud, S. Salinas \& P. Layer 2018. "Geology and radiometric dating of Quaternary monogenetic volcanism in the western Zacapu lacustrine basin (Michoacán, México): implications for archeology and future hazard evaluations", Bulletin of Volcanology, 80(2), 18.

Rojas Rabiela T. 1988. Las siembras de ayer; la agricultura indigena del siglo XVI, Secretaría de Educación Pública, CIESAS, Mexico.

Shoji S., M. Nanzyo \& R. A. Dahlgren 1993. Volcanic Ash Soils: Genesis, Properties and Utilization, Elsevier, Amsterdam.

Siebe C., M.-N. Guilbaud, S. Salinas, P. Kshirsagar, M. O. Chevrel, J. R. de la Fuente, A. Hernández Jiménez, \& L. Godínez 2014. Monogenetic volcanism of the Michoacán-Guanajuato Volcanic Field: Maar craters of the Zacapu basin and domes, shields, and scoria cones of the Tarascan highlands (Paracho-Paricutin region), Pre-meeting field guide for the 5th International Maar Conference, Querétaro, México.

Taladoire E.1989. "Las canchas de juego de pelota de Michoacán (CEMCA : Proyecto Michoacán)" Trace, nº 16, CEMCA, México : 88-99.

Tricart J. 1992. "La Cuenca lacustre de Zacapu: un acertamiento geomorphológico", in Michelet D. (coord.) El Proyecto Michoacán 1983-1987: medio ambiente e introducción a los trabajos arqueológicos, Cuadernos de estudios michoacanos 4, CEMCA, Mexico : 9-52 
Whitmore T. M. \& B. L. Turner II 2001. Cultivated Landscapes of Middle America on the Eve of Conquest, Oxford University Press, Oxford

Yokoyama R., M. Shirasawa \& R. J. Pike 2002. "Visualizing topography by openness: a new application of image processing to digital elevation models", Photogrammetric engineering and remote sensing, 68(3) : 257-66.

Zinck A. J. 2012. Geopedologia. Elementos de geomorfología para estudios de suelos y de riesgos naturales, Faculty of Geo-Information Science and Earth Observation, Enschede. 